\title{
Correction to: Essentials of Marine Biotechnology
}

\section{Correction to:}

Chapter 6 in: S.-K. Kim, Essentials of Marine Biotechnology, https://doi.org/10.1007/978-3-030-20944-5_6

In the original version of the book, belated author corrections have been incorporated and Figure 6.14 has been updated. The current version of the book has been updated with the changes. 